\title{
Verdacht auf sexualisierte Gewalt - was dann?
}

\author{
Access to health services is a fundamental aspect of the right to health. The violation \\ of women's right to health - physically, emotionally, spiritually and socially - inherent in \\ the experience of sexual violence is further compounded when victim/survivors are \\ denied access to gender sensitive sexual violence services. ${ }^{1}$
}

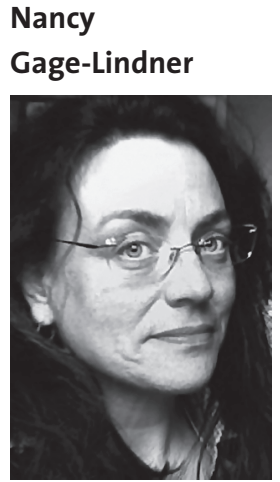

Mitglied der Kommission Gewalt gegen Frauen und Kinder des djb; Leiterin des Referats Prävention und Schutz vor Gewalt im Hessischen Sozialministerium, Wiesbaden.

\section{Problemstellung}

Sexualisierte Gewalt stellt einen massiven Angriff auf die körperliche und seelische Unversehrtheit und eine schwerwiegende Menschenrechtsverletzung dar. Sie kann zu behandlungsbedürftigen Verletzungen körperlicher Art und Folgebeschwerden psychischer Art, bis hin zur Suizidalität, führen. Sie geschieht am ehesten in der Partnerschaft, dort tendenziell mit intensiveren Verletzungen und höherem Gefährdungspotential. Die Häufigkeit ist immens. Nach der repräsentativen Studie zur Gewalt gegen Frauen in Deutschland von Ursula Müller und Monika Schröttle et alia aus 2004 ist davon auszugehen, dass sexualisierte Gewalt gegen Frauen - wie bei der Gewalt gegen Frauen insgesamt - zum ganz überwiegenden Teil von männlichen Beziehungspartnern ausgeübt wird. Fast jede siebte Frau gab an, seit dem 16. Lebensjahr Formen von sexueller Gewalt im strafrechtlichen Sinne erlebt zu haben; hiervon gaben 69 Prozent die eigene Wohnung als Tatort an, 99 Prozent der Täter waren männlich. ${ }^{2}$

Langfristige materielle und psychosoziale Folgen können mit jeder Erfahrung interpersoneller Gewalt einhergehen. Dies gilt in besonderem Maße nach Partnergewalt einschließlich sexualisierter Gewalt. Gekoppelt mit chronischem Verlust an Selbstwertgefühl sind damit auch ein höheres Risiko des Wohnungs- und Arbeitsplatzverlustes, ein sich verschlechterndes Gesundheitsverhalten, Einbußen an Lebensqualität, mithin an Lebensjahren, kausal verbunden. ${ }^{3}$

Dies verlangt nach konsequenter Prävention, die der Verschlechterung des Zustands entgegenwirkt und möglichst der Gewalt auch vorbeugt. ${ }^{4}$ Wer sexualisierte Gewalt erlebt, braucht eine ganzheitliche Versorgung: medizinisch, psychosozial und juristisch. Das schließt eine empfindsam durchgeführte körperliche Untersuchung und Befunderhebung und den Zugang zu einer (sehr komplexen) Spurensicherung mit ein, die für juristische Zwecke zielführend ist. Dies ist nicht ohne Weiteres zu erlangen.

Jeder Schritt hin zu einer Gesundheitspraxis, die eine angemessene, schützende Versorgung von Gewaltbetroffenen tatsächlich erreichen lässt und letztlich eine Stärkung der Patient/innen bewirkt und somit primär präventiv wirkt, ist schon aus pragmatisch-juristischer Sicht dringend notwendig: Zum einen für die Handlungsfähigkeit der Justiz und für den Rechtsbeistand von gewaltbetroffenen Frauen, letztlich aber auch, um der staatlichen Verpflichtung zur Wahrung der Menschenrechte nachzukommen.

In Deutschland können insbesondere die Strafverfolgungsbehörden auf eine über Jahre gewachsene fachliche Spezialisierung verweisen. Durch die Bildung von Fachkommissariaten bei der Polizei und Sonderdezernaten in der Staatsanwaltschaft müsste generell ein hoher Grad an Sensibilisierung gegenüber Gewaltopfern erreicht sein. Auch die Beratungsstellen Frauennotruf und Frauenhäuser gehen zunehmend auf die Strafverfolgungsbehörden zu mit dem Ziel, die psychosoziale Unterstützung engmaschiger zu gestalten. ${ }^{5}$

Nach wie vor fehlt dagegen eine reibungslose Kooperation mit dem Gesundheitssektor. Dabei gaben die für die deutsche Prävalenzstudie befragten Frauen an, dass Ärztinnen und Ärzte für sie die ersten, teils einzigen Ansprechpersonen nach einer Gewalterfahrung sind. ${ }^{6}$

Die Bedeutung der Gewalt an Frauen und die Erfordernisse einer guten Versorgung nach sexualisierter Gewalt insbesondere gewinnt erst jetzt, und

1 Astbury, J., Poster session SVRI: Realizing the right to health: Barriers to access amongst victim/survivors of sexual violence in low income countries, Zugriff 24.3.08, http://www.svri.org/forumabstract.pdf.

2 Müller, U./Schröttle, M. (2004) Lebenssituation, Sicherheit und Gesundheit von Frauen in Deutschland. Eine repräsentative Untersuchung zu Gewalt gegen Frauen in Deutschland. Im Auftrag des Bundesministerium für Familie, Senioren, Frauen und Jugend. Lang- und Kurzfassung: http://www.bmfsfj.de/Kategorien/ Forschungsnetz/forschungsberichte, did $=20560 . \mathrm{html}$ (im Folgenden Müller/Schröttle); vgl. Bericht hierzu Koehler/Gage-Lindner in aktuelle informationen 2005 Heft 2, S. 5 f.; dieser Befund trifft international zu, s.a. Garcia-Moreno, C. et al (2005) WHO Multi-Country Study on Women's Health and Domestic Violence against Women, http://www.who.int/gender/ violence/who_multicountra_study/en/index.html.

3 Müller/Schröttlle; Garcia-Moreno, Fn 2.

4 Vgl.WHO Expert meeting on the primary prevention of intimate partner violence and sexual violence Meeting Report, May 2-3 2007, Zugriff 24.3.08, http://www.who.int/violence_injury_prevention/ violence/activities/intimate/en/index.html.

5 Hier findet ein Paradigmawechsel statt, nachzulesen unter http://www.frauennotrufe.de/dokumente/.

6 Müller/Schröttle, Fn 2, S. 18 (Kurzfassung). 
dies zögerlich, im deutschen Gesundheitswesen an praktischer Relevanz. ${ }^{7}$ Nach wie vor schließt die Gesundheitspolitik eine eigenständige Verantwortung dieses Sektors für die Gewaltprävention - das Ziel der ganzheitlichen Versorgung - als systemfremd aus. ${ }^{8}$

Erste Versuche einer Sensibilisierung und Professionalisierung: In zwölf der sechzehn Bundesländer wurden seit 2000 Anamnese- und Dokumentationsanleitungen veröffentlicht. Vereinzelt sind auch Interventionsvorhaben in Kliniken und Angebote in der Aus-, Weiter- und Fortbildung von Gesundheitsberufen unternommen worden. Der Nachweis der Effektivität und Nachhaltigkeit dieser Initiativen steht noch aus. ${ }^{9}$

\section{Mythos Vergewaltigung}

Schon an der persönlichen Einstellung der beteiligten Berufsgruppen können die besten Absichten scheitern. Jüngste Studien zeigen, dass patriarchalisch geprägte Mythen über die Schuld des Opfers der sexualisierten Gewalt noch lange nicht überwunden sind.

Male dominance and gender inequality can influence the allocation of resources for sexual assault services and, ultimately, the existence, quality and effectiveness of medico-legal evidence. ${ }^{10}$

Ernüchternd ist das Ergebnis der 2007 veröffentlichten Untersuchung über die Bedeutung der rechtsmedizinisch geschulten Spurensicherung bei sexualisierter Gewalt weltweit, die Janice Du Mont und Deborah White im Auftrag der Weltgesundheitsorganisation (WHO) durchführten. Wird der erlebte Übergriff nicht aus Sicht des Opfers nachempfunden, sondern dem Opfer vielmehr das Geschehen prinzipiell angelastet, dann kann auch die scheinbar gründlichste Ermittlung ein Verfahren nicht retten. Fehlt bereits ein Problembewusstsein für die Verfälschung der Sachverhaltsklärung durch Vorurteile gegen Vergewaltigungsopfer und Frauen im Allgemeinen, werden zwangsläufig schwerwiegende Fehler gemacht. So wird nicht selten gezielt der Nachweis gesucht, dass das Geschehen nur mit Zustimmung erfolgt sein könne. ${ }^{11}$ Bis heute bleiben ein faktisch irrelevantes Verhalten vor, während und nach der Tat oder auch die Bekleidung einer Opferzeugin für Verfahrensergebnisse ausschlaggebend. ${ }^{12}$
Für die Gewaltbetroffene bedeutet ein solches sinnentstellendes Ergebnis eine erneute Viktimisierung, die mit schwerwiegenden Folgen verbunden ist. ${ }^{13}$ Nicht ohne Grund besteht bis heute weltweit eine besonders niedrige Bereitschaft zur Strafanzeige bei Sexualdelikten. ${ }^{14}$

Rechtspolitisch ist in Deutschland viel zur Verbesserung der Opferrechte im Strafverfahren getan worden. ${ }^{15}$ Mehr Aufmerksamkeit verdient das Risiko, dass bereits die nicht professionell vorgenommene körperliche Untersuchung und deren Auswertung später durch die Justiz zu einem verfälschenden Verfahrensausgang führen können.

\section{Grundvoraussetzungen einer erfolgreichen Praxis}

Du Mont/White bestätigen allerdings angesichts einiger vielversprechender Praxisbeispiele, dass dort,

7 Hieraus zog die Bundesregierung die Konsequenz, im Aktionsplan II zur Bekämpfung der Gewalt gegen Frauen einen Schwerpunkt auf den Gesundheitssektor zu legen, BT Drs. 16/6584 vom 28.9.07, http://www. bmfsfj.de/bmfsfj/generator/Politikbereiche/ Gleichstellung/frauen-vor-gewalt-schuetzen.html.

8 BR-Drs. 374/07 vom 29.5.07, Stellungnahme der Bundesregierung zu der Entschließung des Bundesrates für eine Ausweitung und Qualifizierung der Früherkennungsuntersuchungen im Sinne des Kindeswohls.

9 Vgl. Bestandsaufnahme im Aktionsplan II, Fn. 7; Blättner, B. et al (2008) Ärzte sollten ganz genau hinsehen, Deutsches Ärzteblatt Heft 1-2. S. A23 ff.; Blättner, B./ Müller, I. (2007) Ergebnisse einer Befragung hessischer Ärztinnen und Ärzte, Hessisches Ärzteblatt Heft 9, S. 565 ff.; Hornberg, C./Schröttle, M. et alia (in Vorbereitung), Expertise für die Erstellung eines Themenheftes der Gesundheitsberichterstattung des Bundes zum Thema „Gesundheitliche Folgen von Gewalt" im Auftrag des Robert-Koch-Instituts.

10 Du Mont, J./White, D. (2007) The uses and impacts of medico-legal evidence in sexual assault cases: A global review. Im Auftrag der Weltgesundheitsorganisation für die Sexual Violence Research Initiative (SVRI), http://www.svri.org/medico.pdf, S. 2, 34 ff.

11 Dto., S. 42 ff. zu Vorurteilen und Sub-Kulturen aller relevanten Professionen.

12 Dto., S. $30 \mathrm{ff}$.

13 Die Folgen der Sekundärviktimisierung sind verschiedentlich untersucht worden, vgl. Müller/Schröttle und Garcia-Moreno, Fn. 2, die französiche Prävalenzstudie ENVEFF von 2002/2003, erörtert in Gage-Lindner, N. (2005) Gewalt gegen Frauen in Frankreich, Streit, S. 12 ff.

14 Vgl. Müller/Schröttle, Garcia-Moreno et al, Du Mont/ White, Fn 2, 10, WHO (2003) Guidelines for medico-legal care for victims of sexual violence, http://www. who.int/violence_injury_prevention/publications/ violence/med_leg_guidelines/en/index.html.

15 S. hierzu Aufsātz von Kräuter-Stockton, S. in diesem Heft S. 78. 
wo frauenfeindliche Einstellungen bewusst überwunden und verlässliche, kooperative Strukturen zwischen Gesundheitssektor, Strafverfolgung und Justiz und dem Hilfesystem etabliert werden, die rechtsmedizinisch geschulte Spurensicherung durchaus zielführend funktionieren kann. ${ }^{16}$

Hierzu gehört solides Wissen aller beteiligten Berufsgruppen über geschlechtsbezogene Gewalt. Günstig ist hierfür als erster Schritt die Beleuchtung der Einstellungen des Personals zu Geschlechtsrollen. Zudem muss die sexualisierte Gewalt entmystifiziert werden, das heißt der Mythos muss ausgeräumt werden, dass die Vergewaltigung zum Beispiel nur durch einen Fremden ausgeübt wird, ein Überfall nur nachts an verlassener Stelle im öffentlichen Raum passiert bzw. nur einer sexuell Unerfahrenen, indes nie einer geschlechtsneutral Bekleideten widerfahren kann.

Außerdem gilt es, institutionelles Wissen zu etablieren, also dafür zu sorgen, dass auch beim Weggang von geschultem Personal das Verhalten der Beschäftigten weiter durch einen verbindlichen Kodex der Institution gesteuert wird. Es hat sich erwiesen, dass das Wissen um die geschlechtsbezogene Gewalt sowohl durch das konstant geführte Fachgespräch als auch durch die obligatorische Verwendung einer standardisierten Anleitung zur Spurensicherung und Befunderhebung nachhaltig erhalten werden kann. ${ }^{17}$

\section{Bemühen um hohe Standards in Hessen}

Gerade mit dem Ziel, den Beitrag der Ärzteschaft und Pflege auf eine standardisierte und opferzentrierte Basis zu stellen, veröffentlichte das Hessische Sozialministerium 2007 die Anleitung „Befunderhebung, Spurensicherung, Versorgung bei Verdacht auf sexualisierte Gewalt“. ${ }^{18}$ Die Veröffentlichung erklärt Schritt für Schritt die sorgfältige Untersuchung und Dokumentation bei sexualisierter Gewalt gegen Heranwachsende und Erwachsene und leitet zur schonenden Ansprache der Opfer und schließlich zur engen Zusammenarbeit mit den lokalen Schutzeinrichtungen an. Die Veröffentlichung bietet auch eine mehrsprachige Orientierung der Gewaltbetroffenen, um eine informierte Entscheidung über jeden Schritt wie auch über die mittel- und langfristige Versorgung zu erleichtern. Der Respekt gegenüber der Patientin und dem Patienten erfordert es, dass dem schonenden Umgang Priorität vor jedem Erkenntnisinteresse gewährt wird, zumal die Untersuchung freiwillig ist, auch wenn sie in polizeilichem Auftrag erfolgt.

Parallel wurde unter Federführung des Hessischen Landeskriminalamts ein Spurensicherungskit auf den letzten Stand der Technik gebracht. Hierfür wurden unter anderem selbsttrocknende Spurenbehälter entwickelt. Die Fertigung war Anfang 2008 abgeschlossen.

Entstanden ist das Konzept in einem interdisziplinären Redaktionsteam, das die Koordinierungsstelle der hessischen Frauennotrufe im Auftrag des Hessischen Sozialministeriums koordiniert hat. Beteiligt waren Rechtsmedizin, Allgemeinmedizin und Gynäkologie, ein Fachkommissariat (Kriminalpolizei), die Leitung der DNA/Spurensicherung beim Landes- kriminalamt, ein Sonderdezernat für Sexualdelikte (Staatsanwaltschaft), in der Nebenklagevertretung und im Strafrecht spezialisierte Fachanwältinnen, weitere Fachkräfte aus Beratungsstellen und die Verfasserin.

Darüber hinaus erfolgte eine Praxiserprobungsphase in klinischer Praxis und bei Pro Familia. Vor Fertigstellung des endgültigen Entwurfs wurde ein Feedback von rund 40 Sachverständigen aus der Rechtsmedizin, Strafverfolgungsbehörden, der Anwaltschaft sowie den psychotherapeutischen, medizinischen und Pflegeberufen berücksichtigt (peer review).

Grundlage der hessischen Veröffentlichung sind jüngste Empfehlungen der WHO für die rechtsmedizinisch geschulte Versorgung der Opfer sexualisierter Gewalt ${ }^{19}$ und des Robert-Koch-Instituts, Schlussfolgerungen aus der ersten multinationalen Untersuchung zu Gewalt gegen Frauen (WHO $2005)^{20}$, praxisbewährte Richtlinien aus dem Ausland und die konkreten Erfahrungen einschlägiger Spezialist/innen.

Die Herausforderung in der praktischen Umsetzung ist jetzt nicht nur die Aneignung der fachgerechten Techniken, die für die diagnostisch tätige Praxis ungewohnt und umständlich erscheinen, sondern auch die Aneignung von Kenntnissen über Ausmaß, Häufigkeit und Folgen der sexualisierten Gewalt. Zudem gilt es, eine verlässliche Struktur zu schaffen, die es ermöglicht, Spurensicherung, Befunderhebung und Versorgung in einem multi-disziplinären Team zu bewerkstelligen und hierauf den Alltag in Arztpraxen und Kliniken auszurichten.

Als ersten Umsetzungsschritt sollen zunächst die Fachkommissariate der hessischen Kriminalpolizei mit Frauennotrufstellen Multiplikatorenteams bilden, um praktikable Wege für die Untersuchungen und Spurensicherung vor Ort auszuloten und möglichst zu vereinbaren. ${ }^{21}$ Multidisziplinäre Fortbildungskonzepte sollen in nächsten Schritten unter Beteiligung von Justiz, Strafverfolgung, Hilfesystem und Rechtsmedizin flankierend realisiert werden. Schließlich wird die Einrichtung von spezialisierten Untersuchungsstellen für Opfer jeglicher Form der interpersonellen Gewalt geprüft, die zudem über überregionale Fortbildungsangebote und Forschung eine landesweite Ausstrahlung entfalten sollen.

16 Du Mont/White, Fn 10, vgl. Zusammenfassung in Box 6.1, S. 50.

17 Dto.

18 http://www.frauennotruf-frankfurt.de/AerztlicheDokumentation.40.o.html.

19 WHO (2003), Fn. 14.

20 Garcia-Moreno, Fn. 2.

21 Die Übertragung des Dortmunder Konzepts für die anonyme Spurensicherung auf Hessen wird (kontrovers!) diskutiert, vgl. Beitrag Harms, P., aktuelle informationen 2007 Heft 3, S. 13 ff. 